\title{
EL ALEMÁN EN LA ENSEÑANZA SECUNDARIA
}

DANIEL OROZCO CORONIL

I.E.S. Jorge Guillen, Torrox (Málaga)

\section{L} a situación de la enseñanza del alemán en los centros públicos de la Secundaria no ha variado mucho en los últimos quince años en Andalucía. Sólo son ocho los centros que imparten esta asignatura como primer, segundo e incluso tercer idioma moderno en nuestra región autónoma. Las autoridades educativas parecen no querer darse cuenta del avance que la lengua alemana está teniendo en el panorama educativo, no sólo en España (como es el caso de Cataluña y Baleares), sino también en el resto de Europa (me remito al artículo de Esther Peralta en nuestra revista Magazin $n^{\circ} 2$ de marzo de 1997).

En innumerables ocasiones miembros de nuestro reducido gremio docente se han reunido con representantes de la

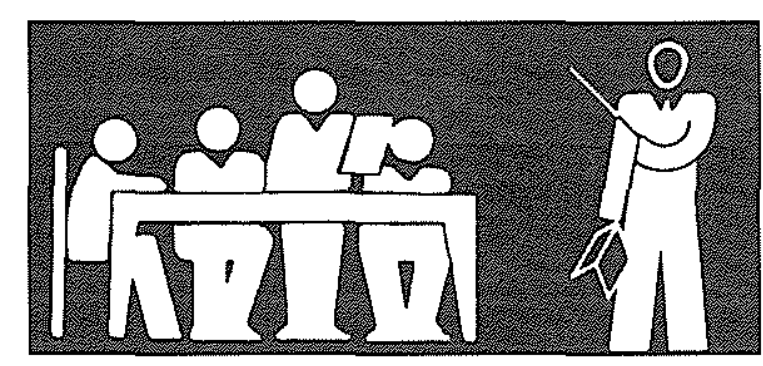

I.F.P. VIRGEN DE LAS NIEVES

CORDOBA: I.E.S. SENECA

SEVILLA: I.E.S. SAN ISIDORO

MALAGA: I.E.S. JORGE GUILLEN (TORROX)

De aqui podemos extraer varias conclusiones, siendo la más obvia la existencia de tres provincias (Jaén, Huelva y Almería) y de una capital de provincia (Málaga) en las que no se enseña alemán en Secundaria. Junto al deseo que todos compartimos de que el alemán se extienda como idioma extranjero en los centros de secundaria de toda Andalucía y de que los institutos anteJunta responsables de Educación aunque, desafortunadamente, estos encuentros han sido siempre infructuosos. Las reivindicaciones de cara a una mayor expansión del alemán en la lista de asignaturas elegibles por los alumnos en Secundaria no han encontrado apoyo por parte de la Administración. Actualmente sólo son cinco las provincias andaluzas que imparten clases de alemán de manera oficial en la ESO, BUP, COU y FP:

CADIZ: I.E.S. COLUMELA

I.F.P. SAN SEVERIANO

I.F.P. ANDRES BENITEZ (Jerez de la Frontera)

GRANADA: I.E.S. PADRE SUAREZ riormente citados dejen de ser los "mirlos blancos" de cada provincia, entendemos que existen graves deficiencias como la de Málaga capital, en la que, a pesar de su enorme potencial turístico, no existe un centro en el que se imparta el alemán.

\section{Turismo}

Asimismo es incomprensible que los destinos turisticos con mayor afluencia de alemanes (Costa del Sol, Costa Tropical en Granada, Costa de la Luz en Huelva y Cádiz, y la Costa almeriense) carezcan de centros docentes en los que los/as alumnos/as puedan ir familiarizándose con la lengua alemana y así poder establecer una mejor comunicación entre los 


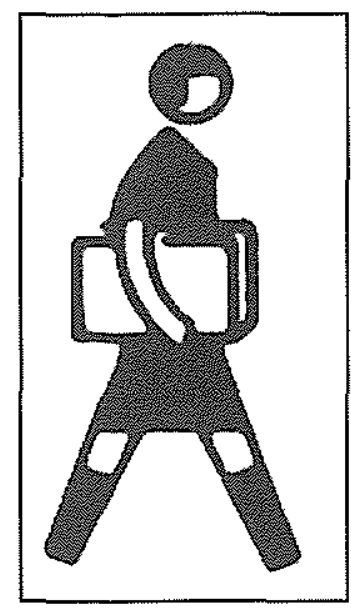

se base principalmente en el turismo se descuida tanto un sector tan importante como el de la enseñanza de lenguas

extranjeros (muchos de ellos residentes fijos) y los nativos del lugar. En pocos lugares del mundo cuya economia

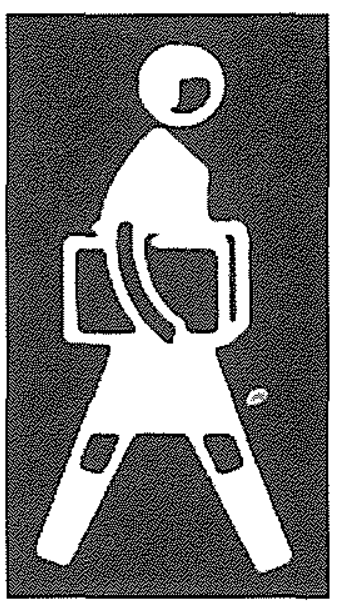

claves para el desarrollo del mismo. Es vergonzoso que el español despierte más interés en los institutos alemanes que a la inversa.

\section{Politica educativa}

De la escuela pública tenemos más datos que de la escuela privada en cuanto a la enseñanza del alemán. No obstante nos consta el aumento de plazas de alemán como optativa de $2^{\circ}$ idioma moderno en centros privados andaluces. Este crecimiento es mayor que en la escuela pública, donde no se convocan oposiciones para Secundaria en la especialidad de alemán desde 1993 y tampoco hay grandes perspectivas de que la situación cambie en un futuro próximo debido, principalmente, a la desastrosa puesta en funcionamiento de la E.S.O. en nuestra Autonomia, asi como al continuo recorte presupuestario en Educación.

Las esperanzas que en un principio suscitó la LOGSE con la implantación de un $2^{\circ}$ idioma moderno obligatorio en la Educación Secundaria se han ido desvaneciendo poco a poco. Todo ha quedado como un bonito sueño nunca hecho realidad. En efecto, las plazas de alemán que deberian haberse creado nunca llegaron siquiera a ser objeto de estudio en la Consejería de Educación y Ciencia de la Junta de Andalucia. El francés, al respecto, se ha afianzado como $2^{\mathrm{a}}$ lengua extranjera sin dar opción

alguna al alemán, por contar desde el principio con un amplísimo respaldo institucional. Lejos de incitar a un polémico y estéril enfrentamiento con nuestros compañeros de francés, creemos que hubiera sido más justo compartir las plazas de Secundaria para el $2^{\circ}$ idioma moderno.

\section{El alemán en la E.S.O.}

Si observamos la distribución de las asignaturas en la E.S.O. y Bachillerato resulta más evidente lo ilógico del sistema educativo por lo que respecta al $2^{\circ}$ idioma moderno (el horario del primer idioma moderno es de 3 horas en todos los cursos excepto en $2^{\circ}$ BACHILLERATO que es de 2 horas ):

\begin{tabular}{|llll|}
\hline $1^{\circ} \mathrm{ESO}:$ & alemán elegible entre otras optativas & $(2 \mathrm{hs} / \mathrm{sem})$ \\
$2^{\mathrm{a} E S O}:$ & $"$ & $"$ & $(2 \mathrm{hs} / \mathrm{sem})$ \\
$3^{\circ} \mathrm{ESO}:$ & $"$ & $"$ & $(2 \mathrm{hs} / \mathrm{sem})$ \\
$4^{\circ} \mathrm{ESO}:$ & $"$ & & $(3 \mathrm{hs} / \mathrm{sem})$ \\
$1^{\circ} \mathrm{BAC:}$ & obligación de escoger un segundo idioma moderno & $(2 \mathrm{hrs} / \mathrm{sem})$ \\
$2^{\circ} \mathrm{BAC:}$ & $"$ & $"$ & $(3 \mathrm{hrs} / \mathrm{sem})$ \\
\hline
\end{tabular}

Al ver este cuadro nos preguntamos, ¿qué ocurre entre un curso y otro? Pues la experiencia nos demuestra que los alumnos se ven por una parte desinformados y perdidos entre el maremágnum de optativas y por otra, observamos la inclinación hacia

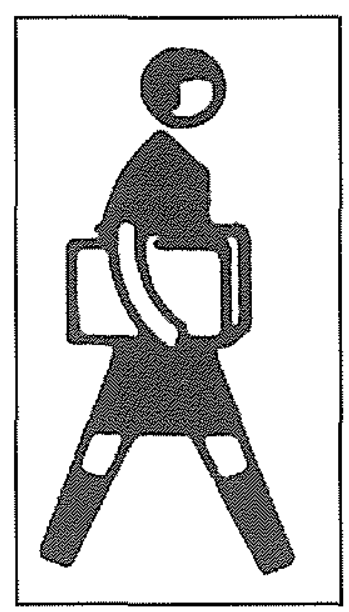

sos de la E.S.O., con alumnos que un año estudiaron alemán 0 francés y que, dependiendo de las numeroaquellas asignaturas que les resultan más fáciles de "superar". El resultado es el enorme baile de listas en los sucesivos cur-
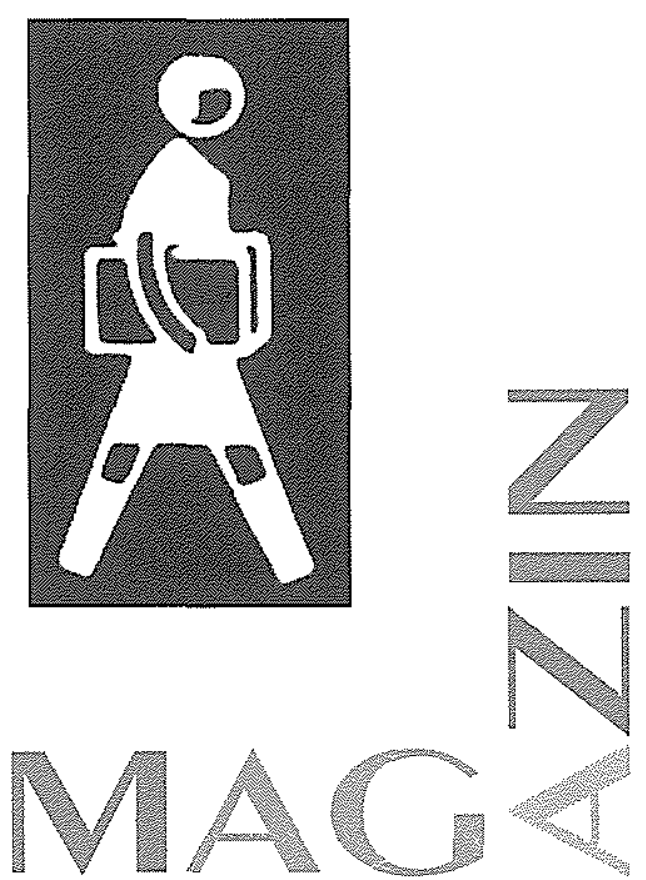


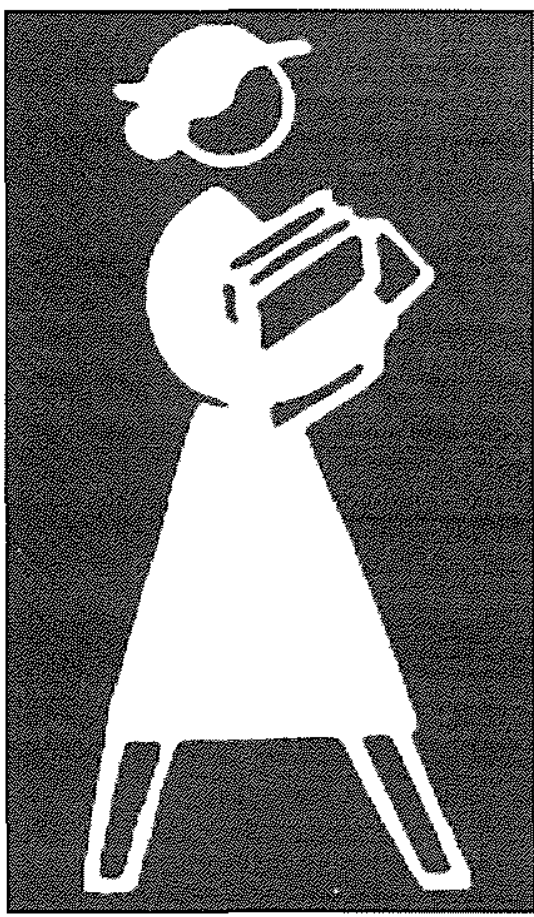

sas optativas de cursos posteriores pudieron seguir cursándolo si es que su combinación de optativas elegidas era compatible con el horario de los diferentes grupos. Es decir, un alumno que haya cursado alemán como optativa en $2^{\circ}$ idioma puede verse privado de él en $4^{\circ} \mathrm{ESO}$, si sus otras optativas no coinciden en horario con las predeterminadas por el centro para el idioma moderno, cosa que ocurre con relativa frecuencia. Los centros tendrian mucho que aportar al respecto, si se acortara la larga y absurda lista de optativas (muchos centros asi lo están haciendo ante las enormes desventajas que ello supone) y se impulsara a los alumnos a elegir el $2^{\circ}$ idioma también en $2^{\circ}, 3^{\circ}$ y $4^{\circ}$ de ESO, excepto a aquellos con graves deficiencias en las áreas de Lengua y Matemáticas consideradas prioritarias en el sistema educativo, a los que se les recomienda la asistencia a Talleres de estas materias.

\section{Los números hablan}

El número de alumnos que en la escuela pública andaluza recibe la enseñanza del alemán en Secundaria es el siguiente (ver cuadro 1):

Como puede observarse, los tres centros (San Isidoro, Padre Suárez y Séneca) mantienen números aceptables de alumnos en primera lengua extranjera, pero sólo porque en sus centros no se ha aplicado la E.S.O. totalmente. El resto de los centros deberia de recibir más ayuda al respecto, potenciando el alemán como primer idioma. Como segunda lengua extranjera vemos que el alemán va ganando poco a poco terreno. Por consiguiente creo que es lícito solicitar desde estas lineas una mayor implantación del alemán en nuestras escuelas, un mayor apoyo por parte de la Administración y un mayor esfuerzo entre todos para que nuestras reivindicaciones se hagan lo más pronto posible realidad. En este proyecto debemos involucrarnos todos, desde las autoridades educativas hasta los profesores de todos los niveles de la enseñanza, pasando por alumnos y APAS ( Asociación de Padres de Alumnos ) y en general todos los germanófilos para que se acabe con el trato injusto que se le ha venido dando al alemán en nuestras escuelas.

D.O.C.

\begin{tabular}{|l|c|c|c|c|c|c|c|c|}
\hline & CA(1) & CA(2) & JERE2 & TORROX & SE & CO & GR(1) & GR(2) \\
\hline cursos & 3 & 6 & 2 & 7 & 7 & 4 & 4 & 4 \\
\hline alumnos (1 $^{\circ}$ id) & - & 5 & - & 4 & 38 & 75 & 49 & - \\
alumnos ( $^{\circ}{ }^{\circ}$ id) & 95 & 53 & 76 & 121 & 55 & - & - & 115 \\
\hline alumnos (total) & 95 & 58 & 76 & 125 & 93 & 75 & 49 & 115 \\
\hline \multicolumn{1}{c}{ TOTAL ANDALUCÍA: 686 ALUMNOS } \\
\hline
\end{tabular}

cuadro 1 


\section{Sprechen Sie Deutsch?}

GOETHE INSTITUT

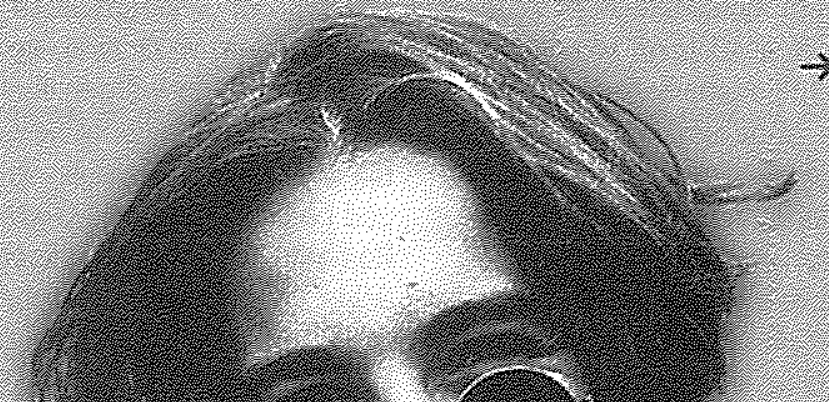

$\rightarrow$ Haga algo para su carrera y aprenda alemáni

Con el institito Goethe en Alemania cursos intensivos, cursos condent sados, cursos especiales Eficacia. segundad, calidad Cenificados de prestinio internacional

\section{Goethe-hstitut Madid}

Tel: $(0034 \cdot 1) 3193235$

Fax: $(0034=1) 31932.46$

internet htip:IIwwwigoethe d:

E-Mali esbogoethe:de
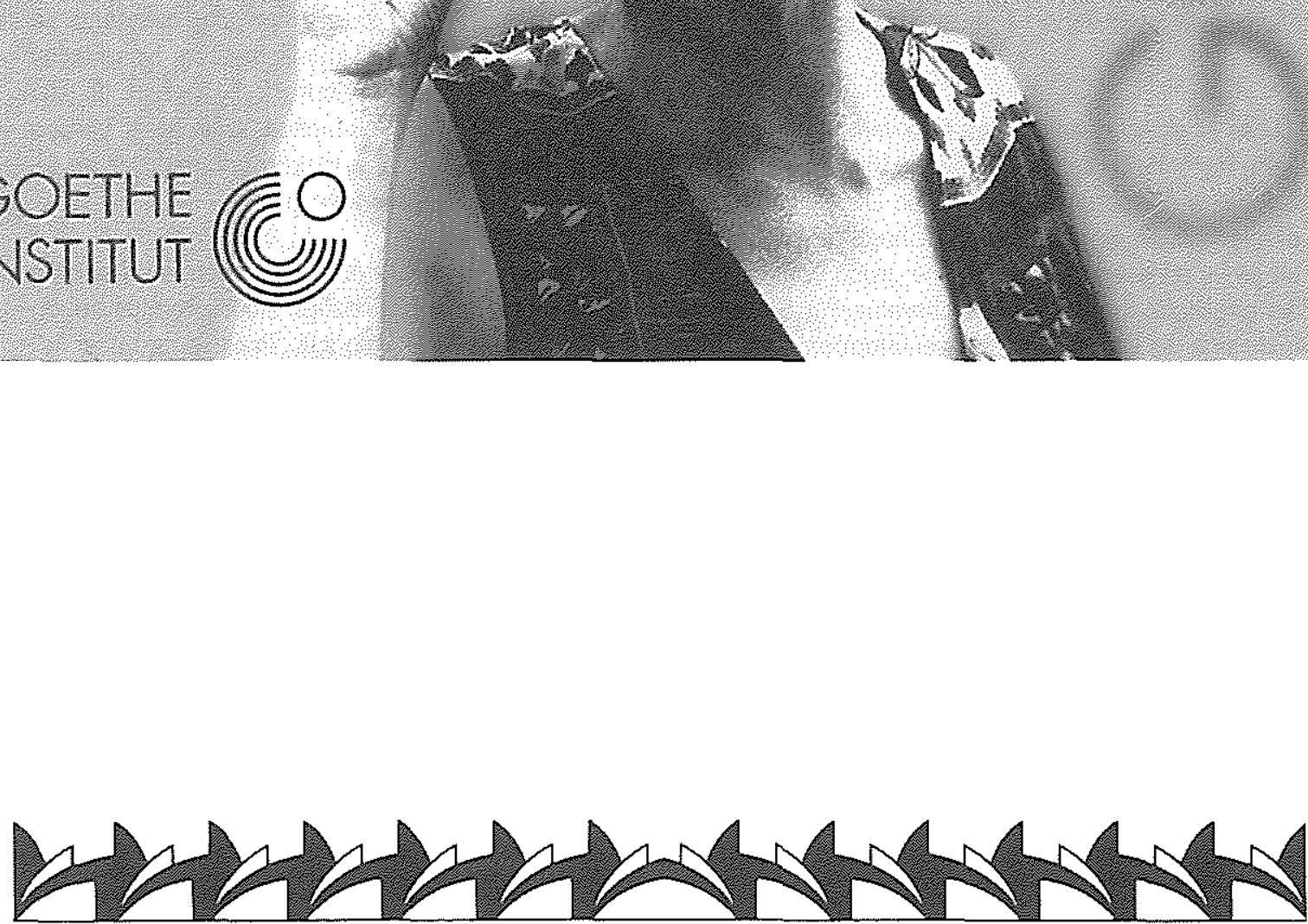